\title{
Elimination of the word length effect by irrelevant sound revisited
}

\author{
SÉBASTIEN TREMBLAY, WILLIAM J. MACKEN, and DYLAN M. JONES \\ Cardiff University, Cardiff, Wales
}

\begin{abstract}
The word length effect refers to the tendency for lists of long words to be recalled less well than lists of short words. Theoretical and empirical objections are raised to a recent claim that irrelevant speech eliminates the word length effect (Neath, Suprenant, \& LeCompte, 1998). A first experiment using a within-subjects design of adequate power $(N=65)$ fails to replicate their finding, showing instead that the word length effect is not differentially eliminated by speech as opposed to tones. In a second experiment, the effect of change (repeated vs. changing sounds) is shown to be additive to the effect of word length for both speech and nonspeech. Irrelevant speech and irrelevant tones have comparable effects on lists of short or lists of long words. These results are at variance with the feature model (e.g., Nairne, 1990).
\end{abstract}

Short-term memory for lists of items, presented either visually or auditorily, is substantially impaired by the mere presence of background sound, either speech or nonspeech, even when the sound has no relevance to the memory task at hand (see, e.g., Colle \& Welsh, 1976; Hanley \& Broadbent, 1987). This so-called irrelevant sound effect is a robust and stable phenomenon, and its disruptive effect reaches up to $50 \%$ (see Ellermeier \& Zimmer, 1997). Under some conditions, the effect of irrelevant sound interacts with other memory phenomena, including articulatory suppression (e.g., Hanley, 1997; Macken \& Jones, 1995), phonological similarity (e.g., Jones \& Macken, 1995; Salamé \& Baddeley, 1986), and the word length effect (Longoni, Richardson, \& Aiello, 1993; Neath, Surprenant, \& LeCompte, 1998). The present work further investigates the interaction of word length and irrelevant sound. More specifically, this study reevaluates a recent claim by Neath et al. that irrelevant speech, but not irrelevant nonspeech, eliminates the word length effect. This pattern of results is at variance with two prominent theories of the irrelevant sound effect-namely, the working memory model (Baddeley \& Hitch, 1974; see also Baddeley, 1992) and the object-oriented episodic record (O-OER) model (Jones, 1993).

To date, two studies have investigated the interaction of word length and irrelevant speech effects. In the first, both effects were shown to be independent (Longoni et al., 1993); however, in a more recent study, the presence of irrelevant speech was shown to eliminate the effect of word

This work was supported in the form of a project grant to D.M.J. from the Defence Evaluation and Research Agency (Farnborough, Hants), Centre for Human Sciences. Thanks are due Eric Farmer and Andrew Belyavin. We thank David Alford and Alaistair Nicholls for critical readings of an earlier draft. Correspondence concerning this article should be addressed to $S$. Tremblay, School of Psychology, Cardiff University, P. O. Box 901, Cardiff CF1 3YG, Wales (e-mail: tremblay@ cardiff.ac.uk). length (Neath et al., 1998). Surprisingly, Neath et al. made no mention of the different pattern of results by Longoni et al. Key features of Neath et al.'s demonstration are subjected to reexamination here because of the divergent results in the two studies.

The word length effect refers to the robust demonstration that recall is worse for lists of long words than that for lists of short words (e.g., Mackworth, 1963). Key functional characteristics of this effect have been disclosed by the way in which a variety of factors modulate the degree to which it is manifest. For example, one determining factor, the time taken to articulate a word, has been highlighted by the interaction of concurrent articulation and word length effects (e.g., Baddeley, Thomson, \& Buchanan, 1975). Concurrent articulation, more commonly referred to as articulatory suppression, involves the repetition of some verbal task-irrelevant item or sequence during the memory task. By preventing rehearsal, articulatory suppression markedly impairs recall performance and has a tendency to eliminate the difference in recall between long-word and short-word lists. The time taken to articulate a word when rehearsing is not the only factor influencing the magnitude of the word length effect. Other time-related factors, such as the output time - that is, pronunciation time at the output stage rather than at rehearsal (e.g., Dosher \& Ma, 1998) - and item-related factors, such as the role of proactive interference (e.g., Nairne, Neath, \& Serra, 1997) or imperfect trace registration (Brown \& Hulme, 1995), also seem to be related to the effect of word length.

In Experiment 1 of Neath et al. (1998), irrelevant speech substantially reduced the advantage of short over long words, for both auditory and visual items, whereas irrelevant tones did not. The authors interpreted this pattern of results as suggesting that the irrelevant speech effect is functionally different than the effect of irrelevant tones. This interpretation is at variance with a substantial amount of research showing functional similarities between ir- 
relevant speech and irrelevant tones. Indeed, both speech and nonspeech act the same way in relation to a variety of phenomena: (1) greater disruption by changing sounds, either tones varying in frequency or speech tokens varying in identity, than by a repeated token (e.g., Jones \& Macken, 1993); (2) stability of the effect over blocks of trials (e.g., Tremblay \& Jones, 1998); (3) insensitivity of the effect to variation in intensity (Tremblay \& Jones, 1999); and (4) the modulating impact of organizational factors, such as streaming by pitch (Jones, Alford, Bridges, Tremblay, \& Macken, 1999).

That irrelevant speech abolishes the word length effect is at variance with the predominant concept of working memory (Baddeley, 1992; Baddeley \& Hitch, 1974). According to the working memory account, the effects of irrelevant speech and word length involve distinct shortterm memory components, and hence, their impact on retention for verbal information should not interact. According to the working memory model, irrelevant speech acts by interfering with the contents of the phonological store, not of the articulatory loop. Given that word length is a property of the loop and not of the store, Neath et al. (1998) take this functional distinction within the working memory model to imply that the model predicts additive effects of irrelevant sound and word length. The O-OER model can be taken to predict the same outcome, but from a different set of premises (see Jones, Beaman, \& Macken, 1996 , for a discussion). Within the O-OER model, it is assumed that interference caused by irrelevant sound is due to a conflict based on the similarity of two concurrent processes-namely, the rehearsal of to-be-recalled items and the perceptual organization of the unattended soundeach involving seriation. Independent effects of word length and irrelevant speech are predicted on the basis of two premises. First, irrelevant sound is assumed to produce its disruption through its action on the process of rehearsal, not on the identity of items in the sequence. Second, given that both long-word lists and short-word lists are subject to rehearsal, they should be equally prone to disruption.

The feature model is quite distinct in both its character and its predictions for the irrelevant speech effect (Nairne, 1990; Neath, in press; Neath et al., 1998). Representations or traces of incoming stimuli are made up of modalitydependent features (physical attributes) and modalityindependent features (e.g., semantic or phonological code). A central notion of the feature model is that recall of an item is a function of a match between its trace in primary memory and a set of relevant traces in long-term memory. According to the feature model, forgetting is mainly due to retroactive interference; there is no decay. The word length effect is explained as a by-product of fragmentation. Longer words have more parts; as the number of parts increases, the likelihood of incorrectly assembling the parts increases. This process is called segment assembly error. Because so many modality-independent features are modified by the presence of irrelevant speech, the effect from a segment assembly error cannot be detected, and so the word length effect is masked. Notably, the irrelevant tones will not mask the word length effect, because they are different from the verbal tokens that make up the to-be-remembered cohort.

The present series of experiments attempts to replicate the findings of Neath et al. (1998) and also to extend the examination of the interaction between word length and irrelevant sound effects. A first experiment compares the impact of irrelevant tones with that of irrelevant speech on the word length effect. A second experiment tests the interaction of variability (repeated vs. changing sounds) and word length.

\section{EXPERIMENT 1}

There seem to be sufficient grounds for doubting that word length and the type of irrelevant sound should interact. We adopted a repeated measures design and reduced the number of conditions in order to increase the power to detect significant effects. Not all the conditions in the Neath et al. (1998) study were replicated; participants undertook the experiment under three auditory conditions (silence, irrelevant speech, and irrelevant tones) and two conditions of word length (short-word lists and longword lists). An articulatory suppression condition was not included, given that the emphasis of this experiment was to test the functional similarity of irrelevant tones and irrelevant speech. Excepting this reduction in the number of conditions and the within-subjects design, the experiment resembles closely Neath et al.'s Experiment 1.

\section{Method}

Participants. Sixty-five students at Cardiff University volunteered to take part in the study in exchange for either course credit or a small honorarium. All the participants reported normal hearing and normal (or corrected-to-normal) vision.

Apparatus and Materials. Items to be recalled were presented serially on the screen of an IBM-compatible PC. Thirty-six lists of 8 short words and 36 lists of 8 long words were constructed from the random arrangement of 81 short words and 81 long words taken from La Pointe and Engle (1990). The arrangement had the constraints that a word could not appear in two consecutive lists and that a word could not be used more than four times. The lists were stored as a text file and presented within a Visual Basic 5.0 environment. During presentation, each word was displayed in the center of the screen in light gray (20-point Times font) on a dark blue background.

Two sequences of irrelevant sound were assembled: one of irrelevant speech and one of irrelevant tones. The irrelevant speech sequence consisted of the word teatime, spoken in a male voice and repeated at a rate of one per second. For the irrelevant nonspeech material, a sequence of alternating tones, whose duration and pitch were based on that of the spoken word teatime, was produced. A first tone, corresponding to tea- was generated at $400 \mathrm{~Hz}$ using SoundEdit Pro 16; the other tone, corresponding to -time, was generated at $250 \mathrm{~Hz}$. A recording was constructed for each condition, delivering $9 \mathrm{sec}$ of sound, presented during the to-be-recalled word sequence. Sound was delivered via headphones from recordings of the sounds held in digital format as .wav files.

Design. A repeated measures design was used, in which each of the 2 (short or long words) $\times 3$ (quiet, irrelevant tones, or irrelevant speech) conditions were presented quasi-randomly, with the constraint that each condition would be presented before any condition was repeated. There were 36 lists of short words and 36 lists of long words; 12 lists of each were presented under each of the three auditory conditions. There were 72 trials in all, 12 for each condition. 
Procedure. The participants were tested individually, seated in a soundproof laboratory approximately $0.5 \mathrm{~m}$ from the computer screen. The participants were also given standard written instructions on the computer screen. The instructions emphasized that the participants should ignore the sound and that they would not be tested on its contents. In each trial, the eight words were presented individually at a rate of one per second (on for $800 \mathrm{msec}$, off for $200 \mathrm{msec}$ ). After the presentation of the whole sequence of eight words, the same items were simultaneously re-presented in 14-point Times font, arranged horizontally at the center of the screen. The left-right order of words was randomized on each trial. The participants were instructed to use the mouse to point and click on the words in the order of their initial sequential presentation. When each word was clicked on, shading was used to signify that the word had been selected. When the response was complete, the participant used the mouse to initiate another trial. The irrelevant sound was played over the headphones during presentation and was switched off automatically during recall. The participants wore the headphones throughout the experimental session. The experiment proper was preceded by a short practice session in quiet. The experiment took some $45 \mathrm{~min}$.

\section{Results and Discussion}

Responses were scored according to a strict serial order recall criterion: The correct item had to be recalled in its presentation position for it to be scored as correct. Figure 1 illustrates percentage of serial recall errors in relation to word length and serial positions for each auditory condition; clearly, both effects are additive. Speech and tones proved to be functionally similar; the presence of irrelevant sound, whether it was speech or nonspeech, did not abolish the effect of word length.

Recall data were then subjected to a 2 (word length) $\times$ 3 (auditory condition) $\times 8$ (serial position) repeated measures analysis of variance (ANOVA). There were significant effects of word length $\left[F(1,64)=58.50, M S_{\mathrm{e}}=\right.$ $10.86, p<.0001]$, auditory condition $[F(2,128)=42.88$, $\left.M S_{\mathrm{e}}=4.39, p<.0001\right]$, and serial position $[F(7,448)=$ $\left.217.05, M S_{\mathrm{e}}=4.13, p<.0001\right]$. The interaction of word length and auditory condition was not significant $[F(2,128)$ $\left.=2.01, M S_{\mathrm{e}}=6.44, p=.14\right]$. The two other interactions, to which no theoretical importance is attached, were significant $(p<.05)$.

The present experiment replicates Longoni et al.'s (1993) results in showing that irrelevant speech does not eliminate the word length effect. We failed to replicate the findings of Neath et al. (1998), despite use of a similar method. A second experiment further investigated the interplay between word length of the to-be-recalled items and irrelevant sound. That irrelevant speech and irrelevant tones act the same way was further tested by contrasting the effect of variability (repeated vs. changing sounds) with that of word length. Although changing state is a key determinant of the irrelevant sound effect, its interaction with the word length effect was not tested in Neath et al.'s study.

Other claims made for the results of Neath et al. (1998) are constrained somewhat by the choice of materials. In particular, the use of the compound word teatime leaves open the possibility that its effects on serial recall can be ascribed either to a repeated single-token sequence or to a repeated two-token sequence. The difference is not in-
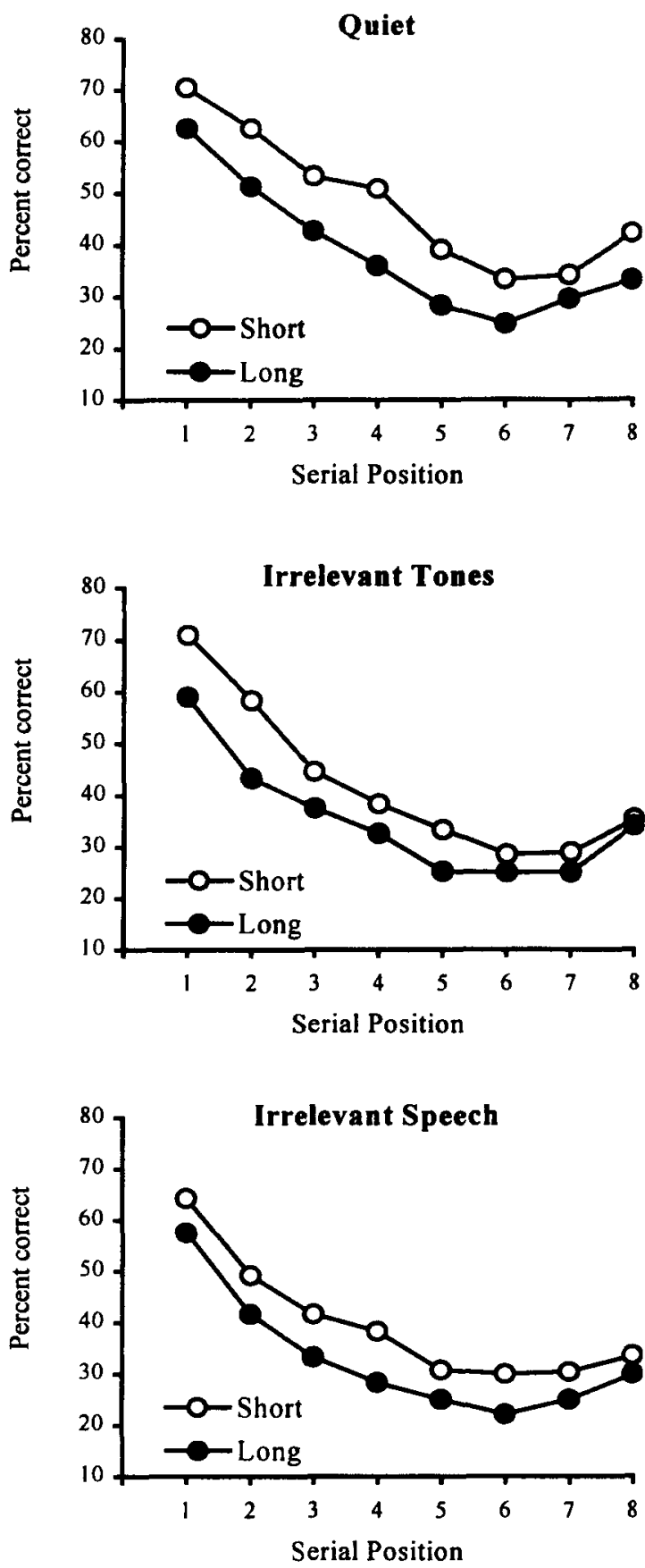

Figure 1. Results from Experiment 1: percentage of short and long words correctly recalled in order as a function of serial position for quiet (top panel), irrelevant tones (middle panel), and irrelevant speech (bottom panel).

consequential. Generally, acoustic or lexical cues may be used to segment an auditory signal. Thereafter, some process calculating mismatch between the acoustic features of successive segmented entities determines the degree of disruption (Tremblay \& Jones, 1998; see Jones et al., 1999, for a discussion). From the standpoint of the O-OER model, teatime can be regarded as a two-part token, in- 
sofar as it can be argued there are sufficient cues to segment the sound into two parts. This doubt weakens the claim made by Neath et al. that the irrelevant sound effect produced by the word teatime is an instance of marked disruption found with repeated tokens (see LeCompte, 1995).

\section{EXPERIMENT 2}

Experiment 1 showed that neither irrelevant speech nor irrelevant tones abolish the recall advantage of short words over long words. In Experiment 2, we examined the possibility of a differential impact of irrelevant speech on the word length effect that depends on whether steady (repeated token law) or changing-state (teatime is considered as two tokens) material is used. The word teatime, claimed to be a repeated token in Neath et al. (1998), is expected to produce greater disruption of ordered recall than does a single repeated token.

\section{Method}

Participants. Thirty-five Cardiff University students took part in return for course credits. All reported normal hearing and normal (or adjusted-to-normal) vision.

Apparatus and Materials. Lists for the memory task were the same as those used in Experiment 1.

Two sequences of irrelevant speech were constructed: one consisted of the word teatime (changing state) and the other one consisted of the monosyllabic word law (steady state), both spoken in a male voice and repeated at a rate of one per second. The recording of those sequences, delivering $9 \mathrm{sec}$ of sound, was presented during the to-be-recalled word sequence.

Design. The design was similar to that used in Experiment 1, except that the auditory conditions consisted of steady and changingstate conditions and a quiet control condition.

Procedure. The procedure was identical to that employed in Experiment 1 .

\section{Results and Discussion}

As is depicted in Figure 2, the results show that the advantage of short words over long words is not abolished by the presence of irrelevant speech. The results were analyzed with a 2 (word length: short and long) $\times 3$ (auditory condition: quiet, repeated token, and changing token) $\times 8$ (serial position) ANOVA. All three main effects were significant [word length, $F(1,34)=63.04, M S_{\mathrm{e}}=8.47$, $p<.0001$; auditory condition, $F(2,34)=20.39, M S_{\mathrm{e}}=$ $6.14, p<.0001$; serial position, $F(7,238)=109.86, M S_{\mathrm{e}}=$ $3.95, p<.0001]$. The interaction of auditory condition and serial position was significant $[F(14,476)=2.86$, $\left.M S_{\mathrm{e}}=1.55, p<.001\right]$. This interaction arises from a progressively less marked degree of disruption by irrelevant sound toward the terminal position. No theory attaches significance to such an interaction; generally, it may be regarded as a scalar effect.

The interaction of auditory condition and word length also reached significance $\left[F(2,68)=3.11, M S_{\mathrm{e}}=5.06\right.$, $p=.05]$. The interaction of word length and auditory condition possibly was due to the disruptive effect of a repeated token relative to quiet when short words had to be recalled or to the slight reduction of the word length
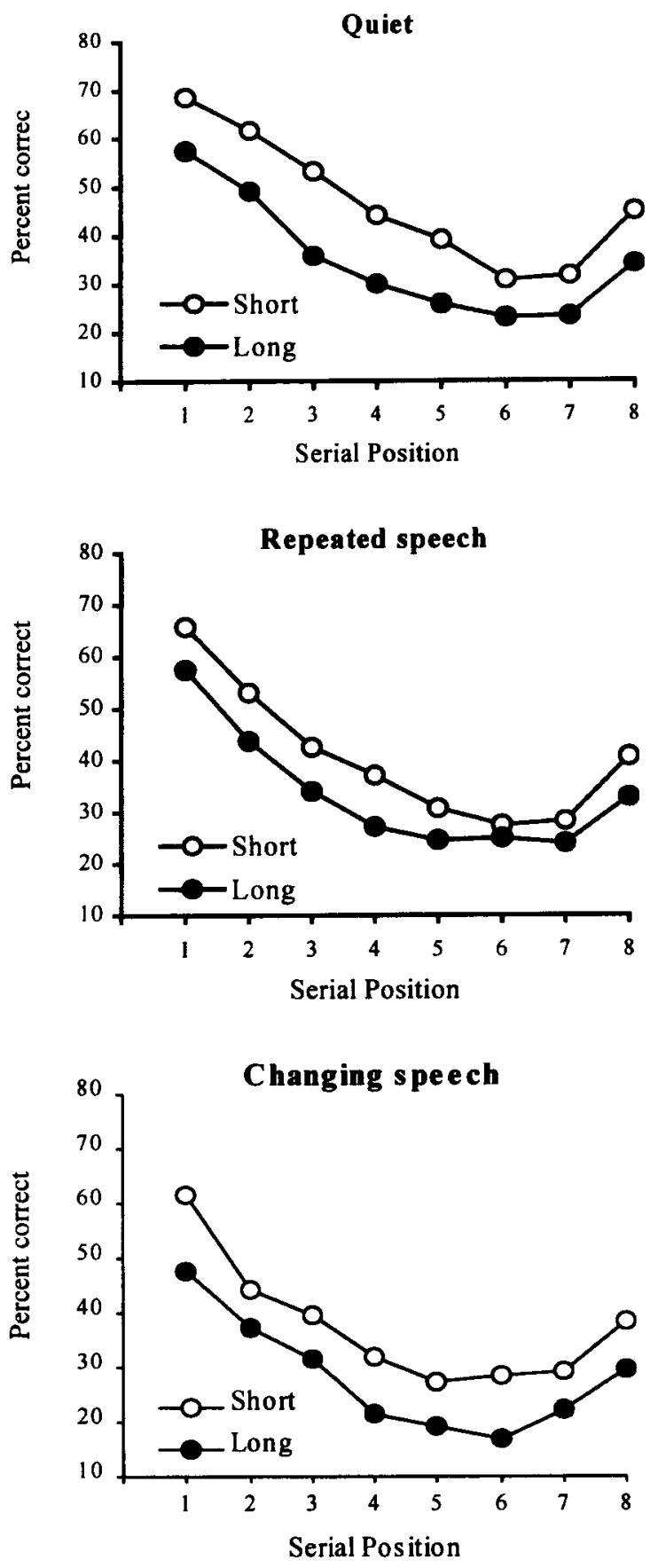

Figure 2. Results from Experiment 2: Percentage of short and long words correctly recalled in order as a function of serial position for quiet (top panel), repeated speech (middle panel), and changing speech (bottom panel).

effect in the presence of irrelevant speech. No other interactions reached significance. Again, irrelevant speech did not eliminate the effect of word length; long words were less well recalled than short words, whether repeated or changing-state stimuli were used as irrelevant speech. As has been demonstrated in previous studies 
(e.g., Tremblay \& Jones, 1998), two tokens are sufficient to produce increased disruption of recall in relation to a repeated token.

\section{EXPERIMENT 3}

Experiment 3 further examined the functional similarity between irrelevant speech and irrelevant tones by attempting to replicate the pattern of results obtained in Experiment 2 , but this time, by using tones instead of speech.

\section{Method}

Participants. Fifty students took part in return for either a small honorarium or course credit. All reported normal hearing and normal (or adjusted-to-normal) vision.

Apparatus and Materials. Lists for serial recall were the same as those used in the previous experiments.

For the irrelevant nonspeech, one sequence was identical to the nonspeech one used in Experiment 1; the other sequence was made up of the repetition of a single tone (one of the two tones that mimic the speech tokens teatime). The three auditory conditions were identical to those contrasted in Experiment 2, except that tones were used instead of speech tokens.

The design and procedure were identical to those used in Experiments 1 and 2 .

\section{Results and Discussion}

Figure 3 shows percentage of short and long words correctly recalled in order as a function of both serial position and auditory condition. These data were analyzed in the same way as in the two previous experiments, by using a $2 \times 3 \times 8$ ANOVA. There were significant effects of word length $\left[F(1,49)=99.86, M S_{\mathrm{e}}=10.54, p<\right.$ $.0001]$, of auditory condition $\left[F(2,98)=18.13, M S_{\mathrm{e}}=\right.$ $4.41, p<.0001]$, and of serial position $[F(7,343)=200.54$, $\left.M S_{\mathrm{e}}=4.33, p<.0001\right]$. There was no interaction between word length and auditory condition $[F(2,98)=$ $\left.2.46, M S_{\mathrm{e}}=3.69, p=.09\right]$. The interactions between serial position and both word length $[F(7,343)=9.75$, $\left.M S_{\mathrm{e}}=1.83, p<.0001\right]$ and auditory condition $[F(14,686)$ $\left.=2.68, M S_{\mathrm{e}} \doteq 1.48, p<.001\right]$ were significant. The threeway interaction was not significant $(p=.22)$.

\section{GENERAL DISCUSSION}

The results are in line with what would be expected from both the working memory model and the O-OER model, in terms of the independent effects of irrelevant sound and word length. As was first shown by Longoni et al. (1993), the word length effect, although slightly reduced, is not abolished by the presence of irrelevant speech. Two findings are at variance with the feature model: first, irrelevant sound (speech and nonspeech) does not eliminate the recall advantage of short words over long words; second, the effect of irrelevant tones seems functionally similar to that of irrelevant speech.

In addition to not being replicated, the results of Neath et al. (1998) are also not internally consistent. If, as they suppose, "the irrelevant speech manipulation results in a memory trace that includes some of the features of the irrelevant item that the subject is hearing" and if this "in-
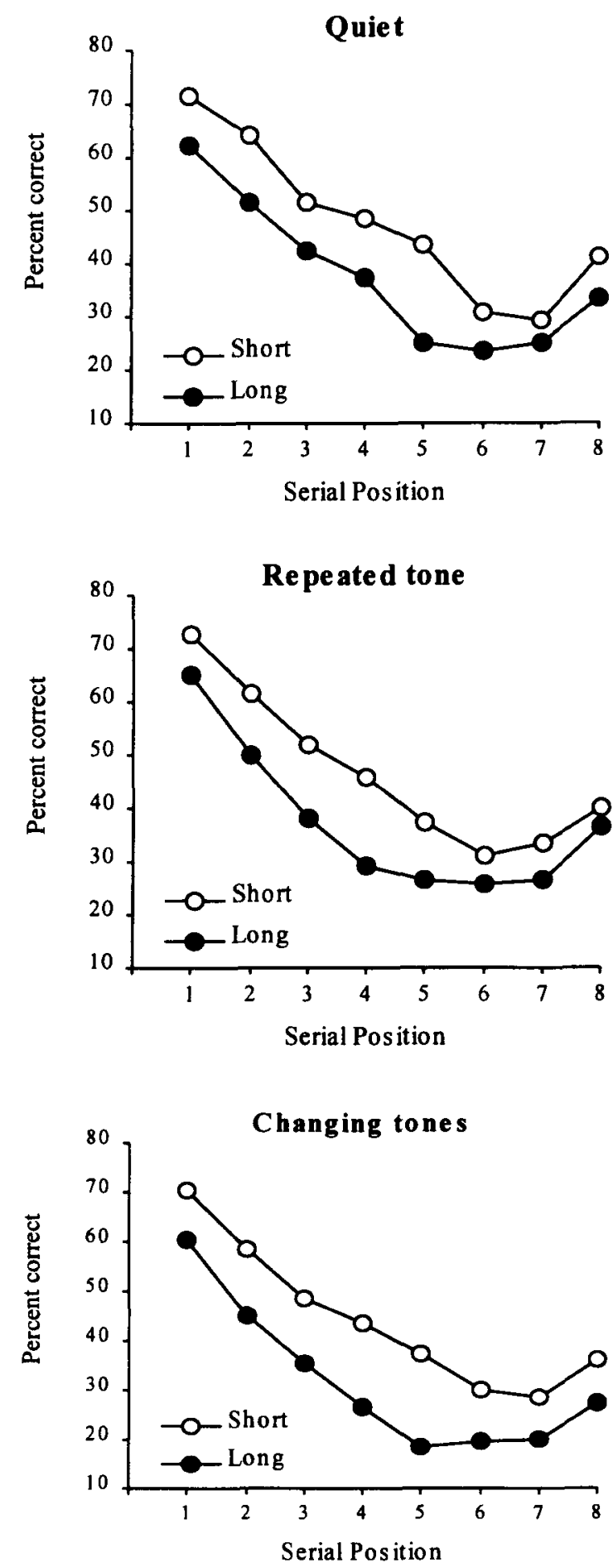

Figure 3. Results from Experiment 3: Percentage of short and long words correctly recalled in order as a function of serial position for quiet (top panel), repeated tone (middle panel), and changing tones (bottom panel).

creases the likelihood of a mismatch, and thereby reduces recall" (p. 345), their finding that tones and speech produce roughly similar effects seems counter to logic. In their Experiment 1, proportions of words correctly recalled 
were .527 with quiet, but, notably, .454 and .456 with tones and speech, respectively (see Neath et al., 1998, p. 346). One might expect that if the features of tones were incorporated into the features of an item, they would remain relatively more distinct than if speech features were incorporated within an item. Clearly, because of the similarity of action of tones and speech, this cannot be the case. In addition, their results support the claim made by Jones and Macken (1993) for the equipotentiality of speech and tones.

The differences that could plausibly have been responsible for the discrepancies between Neath et al.'s (1998) findings and those of Longoni et al. (1993) are related to one or a combination of task variables: the number of items in the set of to-be-remembered words, list length, and the response mode (order reconstruction vs. written serial recall). Set size has already been shown to modulate the interaction of articulatory suppression and word length (Lapointe \& Engle, 1990), but both reconstruction of order and serial recall tasks are similarly susceptible to irrelevant sound, articulatory suppression, and word length effects. The sole difference between Neath et al.'s Experiment 4 and Longoni et al.'s Experiment 3 is list length. However, the present replication of Longoni et al.'s finding - that is, that the word length effect is not abolished by irrelevant speech - with a method similar to that of Neath et al. suggests that the contribution of task variables is unlikely.

It is worth noting that even for reconstruction of order, which involves order information but a minimal load on item information, a substantial effect of irrelevant sound was revealed in the present work and also in Neath et al.'s (1998) study. This result does not seem to support the feature model prediction that disruption is on item identity. In sum, the results reported here cast some doubts on the interactions of word length and irrelevant speech effects found by Neath et al. The elimination of the word length effect by the presence of irrelevant speech formed a central plank of the feature model, but the present study, as well as earlier findings by Longoni et al. (1993), weakens the strength of the model.

\section{REFERENCES}

BADDELEY, A. [D.] (1992). Is working memory working? The fifteenth Bartlett Lecture. Quarterly Journal of Experimental Psychology, 44A, 1-31.

Baddeley, A. D., \& Hitch, G. J. (1974). Working memory. In G. H. Bower (Ed.), The psychology of learning and motivation (Vol. 8, pp. 47-90). New York: Academic Press.

Baddeley, A. D., Thomson, N., \& Buchanan, M. (1975). Word length and the structure of short-term memory. Journal of Verbal Learning \& Verbal Behavior, 14, 575-589.

Brown, G. D. A., \& Hulme, C. (1995). Modeling item length effects in memory span: No rehearsal needed? Journal of Memory \& Language, 34, 594-621.

COLLE, H. A., \& WELSH, A. (1976). Acoustic masking in primary memory. Journal of Verbal Learning \& Verbal Behavior, 15, 17-31.

Dosher, B. A., \& MA, J. J. (1998). Output loss or rehearsal loop? Outputtime versus pronunciation-time limits in immediate recall for forgetting- matched materials. Journal of Experimental Psychology: Learning, Memory, \& Cognition, 24, 316-335.

ELlermeiER, W., \& Zimmer, K. (1997). Individual differences in susceptibility to the "irrelevant speech" effect. Journal of the Acoustical Society of America, 102, 2191-2199.

HANLEY, J. R. (1997). Does articulatory suppression remove the irrelevant speech effect? Memory, 5, 423-431.

HANLEY, J. R., \& BroadBENT, C. (1987). The effect of unattended speech on serial recall following auditory presentation. British Journal of Psychology, 78, 287-297.

JONES, D. M. (1993). Objects, streams, and threads of auditory attention. In A. D. Baddeley \& L. Weiskrantz (Eds.), Attention: Selection, awareness and control (pp. 85-104). Oxford: Oxford University Press, Clarendon Press.

Jones, D. M., Alford, D., Bridges, A., Tremblay, S., \& Macken, W. J. (1999). Organizational factors in selective attention: The interplay of acoustic distinctiveness and auditory streaming in the irrelevant sound effect. Journal of Experimental Psychology: Learning, Memory, \& Cognition, 25, 464-473.

Jones, D. M., Beaman, C. P., \& Macken, W. J. (1996). The objectoriented episodic record model. In S. Gathercole (Ed.), Models of short-term memory (pp. 209-237). London: Erlbaum.

JONES, D. M., \& MACKEN, W. J. (1993). Irrelevant tones produce an irrelevant speech effect: Implications for phonological coding in working memory. Journal of Experimental Psychology: Learning, Memory, \& Cognition, 19, 369-381.

JONES, D. M., \& MACKEN, W. J. (1995). Phonological similarity in the irrelevant speech effect: Within- or between-stream similarity? Journal of Experimental Psychology: Learning, Memory, \& Cognition, 21, 103-115.

LA Pointe, L. B., \& Engle, R. W. (1990). Simple and complex word spans as measures of working memory capacity. Journal of Experimental Psychology: Learning, Memory, \& Cognition, 16, 1118-1133.

LECOMPTE, D. C. (1995). An irrelevant speech effect with repeated and continuous background speech. Psychonomic Bulletin \& Review, 2, 391-397.

Longoni, A. M., Richardson, J. T. E., \& Aiello, A. (1993). Articulatory rehearsal and phonological storage in working memory. Memory \& Cognition, 21, 11-22.

MACKEN, W. J., \& JONES, D. M. (1995). Functional characteristics of the inner voice and the inner ear: Single or double agency? Journal of Experimental Psychology: Learning, Memory, \& Cognition, 21, 436-448.

MACKWORTH, J. F. (1963).The duration of the visual image. Canadian Journal of Psychology, 17, 62-81.

NAIRNE, J. S. (1990). A feature model of immediate memory. Memory \& Cognition, 18, 251-269.

Nairne, J. S., Neath, I., \& Serra, M. (1997). Proactive interference plays a role in the word length effect. Psychonomic Bulletin \& Review, 4, 541-545.

NEATH, I. (in press). Modeling the effects of irrelevant speech on memory. Psychonomic Bulletin \& Review.

Neath, I., Surprenant, A. M., \& LeCompte, D. C. (1998). Irrelevant speech eliminates the word length effect. Memory \& Cognition, 26, 343-354.

Salamé, P., \& Baddeley, A. (1986). Phonological factors in STM: Similarity and the unattended speech effect. Bulletin of the Psychonomic Society, 24, 263-265.

Tremblay, S., \& Jones, D. M. (1998). The role of habituation in the irrelevant sound effect: Evidence from the effects of token set size and rate of transition. Journal of Experimental Psychology: Learning, Memory, \& Cognition, 24, 659-671.

TREMBLAY, S., \& JONES, D. M. (1999). Change of intensity fails to produce an irrelevant sound effect: Implications for the representation of unattended sound. Journal of Experimental Psychology: Human Perception \& Performance, 25, 1005-1015.

(Manuscript received April 12, 1999; revision accepted for publication July 30 , 1999.) 